\title{
High serum granulocyte-colony stimulating factor characterises neutrophilic COPD exacerbations associated with dysbiosis
}

\author{
Arindam Chakrabarti ${ }^{1}$, Jordan S. Mar ${ }^{1}$, David F. Choy ${ }^{1}$, Yi Cao ${ }^{2}$, Nisha Rathore ${ }^{1}$, Xiaoying Yang ${ }^{3}$, \\ Gaik W. Tew ${ }^{4}$, Olga Li ${ }^{4}$, Prescott G. Woodruff ${ }^{5}$, Christopher E. Brightling ${ }^{6}$, Michele Grimbaldeston ${ }^{4}$, \\ Stephanie A. Christenson ${ }^{5}$, Mona Bafadhel $\mathbb{1}^{7}$ and Carrie M. Rosenberger (1) ${ }^{1}$
}

${ }^{1}$ Biomarker Discovery OMNI, Genentech Inc., South San Francisco, CA, USA. ${ }^{2}$ Bioinformatics, Genentech Inc., South San Francisco, CA, USA. ${ }^{3}$ Biostatistics, Genentech Inc., South San Francisco, CA, USA. ${ }^{4}$ OMNI Biomarker Development, Genentech Inc., South San Francisco, CA, USA. ${ }^{5}$ Dept of Medicine, UCSF, San Francisco, CA, USA. ${ }^{6}$ Dept of Respiratory Sciences, University of Leicester, Leicester, UK. ${ }^{7}$ Nuffield Dept of Medicine, University of Oxford, Oxford, UK.

Corresponding author: Carrie M. Rosenberger (rosenberger.carrie@gene.com)

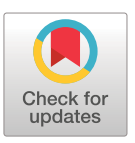

Copyright $\odot$ The authors 2021

This version is distributed under the terms of the Creative Commons Attribution NonCommercial Licence 4.0. For commercial reproduction rights and permissions contact permissions@ersnet.org

This article has supplementary material available from openres.ersjournals.com

Received: 10 Nov 2020 Accepted: 9 March 2021

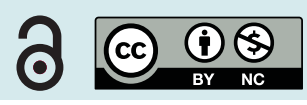

Shareable abstract (@ERSpublications)

Noninvasive biomarkers to characterise \#AECOPD subtypes are limited. High serum G-CSF enriches for COPD exacerbations associated with bacterial infection and neutrophilic inflammation preceded by lung microbial dysbiosis. https://bit.ly/3rck3M6

Cite this article as: Chakrabarti A, Mar JS, Choy DF, et al. High serum granulocyte-colony stimulating factor characterises neutrophilic COPD exacerbations associated with dysbiosis. ERJ Open Res 2021; 7 : 00836-2020 [DOI: 10.1183/23120541.00836-2020].

\section{Abstract}

Introduction COPD exacerbations are heterogeneous and can be triggered by bacterial, viral, or noninfectious insults. Exacerbations are also heterogeneous in neutrophilic or eosinophilic inflammatory responses. A noninvasive peripheral biomarker of COPD exacerbations characterised by bacterial/ neutrophilic inflammation is lacking. Granulocyte-colony stimulating factor (G-CSF) is a key cytokine elevated during bacterial infection and mediates survival, proliferation, differentiation and function of neutrophils.

Objective We hypothesised that high peripheral G-CSF would be indicative of COPD exacerbations with a neutrophilic and bacterial phenotype associated with microbial dysbiosis.

Methods Serum G-CSF was measured during hospitalised exacerbation (day 0 or D0) and after 30 days of recovery (Day30 or D30) in 37 subjects. In a second cohort, serum and sputum cytokines were measured in 59 COPD patients during stable disease, at exacerbation, and at 2-weeks and 6-weeks following exacerbation.

Results Serum G-CSF was increased during exacerbation in a subset of patients. These exacerbations were enriched for bacterial but not viral or type-2 biologies. The median serum G-CSF level was 1.6-fold higher in bacterial exacerbation compared to nonbacterial exacerbation $\left(22 \mathrm{pg} \cdot \mathrm{mL}^{-1}\right.$ versus $13 \mathrm{pg} \cdot \mathrm{mL}^{-1}$, $\mathrm{p}=0.0007$ ). Serum G-CSF classified bacterial exacerbations with an area under the curve (AUC) for the receiver operating characteristic (ROC) curve equal to 0.76. Exacerbations with a two-fold or greater increase in serum G-CSF were characterised by neutrophilic inflammation, with increased sputum and blood neutrophils, and high sputum interleukin (IL)-1 $\beta$, IL-6 and serum amyloid A1 (SAA1) levels. These exacerbations were preceded by dysbiosis, with decreased microbiome diversity and enrichment of respiratory pathogens such as Haemophilus and Moraxella. Furthermore, serum G-CSF at exacerbation classified neutrophilic-dysbiotic exacerbations (AUC for the ROC curve equal to 0.75).

Conclusions High serum G-CSF enriches for COPD exacerbations characterised by neutrophilic inflammation with underlying bacterial dysbiosis.

\section{Introduction}

Acute exacerbations of COPD (AECOPDs) decrease patient quality of life, contribute to progressive airway function decline and can be fatal [1, 2]. Exacerbations are defined by an acute worsening of respiratory function leading to therapeutic intervention with antibiotics or corticosteroids [2]. There have been multiple recent efforts to define exacerbations more precisely by their molecular features, which has revealed 
heterogeneity in this key clinical event [3-5]. The identification of biomarkers to classify exacerbations is a step towards developing a mechanistic understanding of the underlying inflammatory aetiology, which is needed for the development of targeted therapies.

Unsupervised clustering analysis has previously identified four exacerbation phenotypes: bacterial, viral, eosinophilic and pauci-inflammatory. These clusters were defined by pathogens, eosinophils or little inflammation, respectively, in sputum collected during exacerbation [3]. The most sensitive and specific inflammatory biomarkers of these exacerbation phenotypes were sputum interleukin (IL)-1 $\beta$ (bacterial-predominant), serum chemokine (C-X-C motif) ligand 10 (CXCL10) (also known as interferon (IFN)- $\gamma$ induced protein-10 (IP-10), viral-predominant) and the percentage of blood eosinophils (eosinophil-predominant). A similar clustering approach in COPD and asthma patients that did not include microbiological information yielded a neutrophil-predominant cluster containing features attributed to both bacterial and viral exacerbations: high sputum percentage of neutrophils, IL-1 $\beta$, tumour necrosis factor- $\alpha$ (TNF- $\alpha$ ), IL-8 (bacterial) and serum IP-10 (viral) [4]. The subset of exacerbations characterised by high sputum IL-1 $\beta$ and the presence of pathogenic bacteria such as Haemophilus influenzae are also characterised by increased sputum levels of the neutrophil-promoting cytokines granulocyte-colony stimulating factor (G-CSF) and IL-6 [6]. Neutrophils are the predominant inflammatory cell in respiratory samples taken during bacterial and viral exacerbations in many patients, and are a potential therapeutic target [7, 8]. Sputum neutrophil elastase activity is a biomarker for bacterial exacerbation [9], an aetiology accounting for approximately half of COPD exacerbations [3]. A recent report further subdivided neutrophilic COPD based on the abundance of Haemophilus [10]. Since sputum cannot be produced by all patients and is not routinely collected during exacerbations, the present study focuses on identifying a peripheral marker that can be indicative of a COPD exacerbation phenotype associated with underlying airway dysbiosis (with overabundance of potentially pathogenic airway bacteria and high sputum neutrophils).

We hypothesised that peripheral G-CSF could be a biomarker for a neutrophilic COPD phenotype and would enrich for bacterial/neutrophilic exacerbations. Serum G-CSF correlates with airway G-CSF, which is the best noninvasive biomarker of lung neutrophils in the bronchoalveolar lavage fluid of patients with acute respiratory distress syndrome [11-13]. Here, we show that increased serum G-CSF classifies COPD exacerbations associated with airway dysbiosis enriched for potentially pathogenic bacteria and a neutrophil-predominant inflammatory response.

Methods

Study populations

Clinical studies were approved by the relevant ethics review committees (the ethics number for the MRC BEAT:COPD study is 07/H0406/157).

\section{The LEUKO cohort}

Biomarkers were measured in 54 samples at time of exacerbation (Day0 or D0) and 52 samples during recovery (Day30 or D30), along with 37 paired measurements from a double-blind, placebo-controlled, parallel group study of COPD subjects (recorded as NCT00493974 at ClinicalTrials.gov [14]) who were randomised to receive either zileuton tablets $(600 \mathrm{mg}$ ) or placebo tablets (taken orally, 4 times per day, for 14 days). Inclusion criteria included an admitting diagnosis of AECOPD (defined as an acute increase in dyspnoea, sputum volume and/or sputum purulence, without other attributable cause), a smoking history of $\geqslant 10$ pack-years, a value for forced expiratory volume in $1 \mathrm{~s}\left(\mathrm{FEV}_{1}\right)<60 \%$ of predicted at time of inclusion, or an inability to perform spirometry due to dyspnoea.

\section{The MRC cohort}

Biomarkers were measured in 74 exacerbation samples from 59 subjects, with 56 paired serum and sputum measurements (supplementary table S1), along with preceding stable and post-exacerbation samples from a prospective observational study [3]. Inclusion criteria included physician-diagnosed COPD, a post-bronchodilator $\mathrm{FEV}_{1}$ /forced vital capacity (FVC) ratio of less than 0.7 and one or more exacerbation in the preceding 12 months. Samples were collected every 3 months during stable disease for 1 year. Baseline and stable visits were 8-weeks free of an exacerbation that required treatment with oral corticosteroids or antibiotics. Samples were collected during exacerbation and at 2-week and 6-week follow-up visits. Subjects received standard of care oral steroid and/or antibiotic treatments for exacerbations [3]. 


\section{Classification of exacerbation subtype}

Bacteria-, virus- and sputum eosinophil-associated COPD exacerbations are defined according to the original publication from the MRC cohort [3]. Briefly, bacterial exacerbations were defined as having a positive bacterial pathogen on routine culture (H. influenzae, Moraxella catarrhalis, Streptococcus pneumoniae, Staphylococcus aureus or Pseudomonas aeruginosa) and/or a total aerobic colony forming unit (CFU) count of $10^{7}$ or greater. Viral exacerbations were defined as having a positive sputum viral PCR test. Eosinophilic exacerbations were defined by the presence of $>3 \%$ non-squamous cells following sputum cytospin slide preparation. Neutrophilic COPD exacerbations were classified based on a sputum neutrophil count of $\geqslant 61 \%$ at exacerbation and were subdivided by the combined relative abundance (RA) of Haemophilus and Moraxella (dysbiotic $>0.41$ and balanced $\leqslant 0.41$ ) as defined in [10].

\section{Measurements of biomarkers and statistical methods}

Serum and sputum biomarkers were measured using the multiplex enzyme-linked immunosorbent assay (ELISA) platform, including Luminex and as previously described [3]. Statistical analyses were performed using Prism version 8 (GraphPad Software, La Jolla, CA, USA) or the R statistical software package (The R Project for Statistical Computing, www.r-project.org). Biomarker levels were log transformed and median values with 95\% confidence intervals (CIs) were shown. Statistical significance between exacerbation and recovery or preceding stable visits was calculated by the Wilcoxon matched-pairs signed-rank test. Statistical significance between G-CSF high versus G-CSF low groups, or exacerbations \pm bacterial infection, was calculated using the Mann-Whitney U-test. Area under the curve (AUC) for the receiver operating characteristic (ROC) curves was calculated for bacteria-associated versus non-bacteria-associated exacerbations. Correlation heatmaps and the pairwise Spearman correlation coefficients were computed using the "cor" command in R and plots were generated using the "ggplot2" and "ggpubr" $\mathrm{R}$ packages.

\section{SV3-V5 rRNA sequence data processing}

Raw sequence data was obtained from the NCBI Sequence Read Archive database under accession number SRP065072 and processed using QIIMEv1.9 [15]. Raw sequence data was first demultiplexed and quality filtered, keeping reads between 200-1000 bp with a minimum average q-score of greater than 29 and with zero ambiguous base calls. High quality reads were then binned into operational taxonomic units (OTUs) at $>97 \%$ sequence identity using sortmerna and the Greengenes bacterial rRNA database (v13_8) for closed OTU picking, followed by open OTU picking using sumaclust [16]. Chimeric OTUs were removed with ChimeraSlayer (http://microbiomeutil.sourceforge.net/). Chimera-free OTU abundance tables were then rarefied to 4233 reads per sample to account for variable sequencing depths.

\section{Microbiome analysis}

Both $\alpha$ - and $\beta$-diversity measures were calculated in QIIMEv1.9. All statistical analysis was conducted in R. A Wilcoxon rank-sum test was performed to compare $\alpha$-diversity between exacerbation types. Bray-Curtis distance matrices were visualised via non-metric multi-dimensional scaling (NMDS), while permutational multivariate analysis of variance (PERMANOVA) was used to determine the statistical relationships between metadata (i.e. the experimental group) and bacterial microbiota composition in $\mathrm{R}$ using the "vegan" package (https://github.com/vegandevs/vegan). To identify significantly enriched or depleted bacterial taxa in relevant experimental groups, the R package "DESeq2" was used as described by McMurdie et al. [17]. Resulting p-values were adjusted for false discoveries using the Benjamini-Hochberg correction [18].

\section{Human bronchial epithelial cell culture and treatment}

Normal bronchial epithelial cells (Lonza, Basel, Switzerland) were grown and differentiated at the air-liquid interface prior to treatment with recombinant human IL-1 $\beta\left(10 \mathrm{ng} \cdot \mathrm{mL}^{-1}\right)(\mathrm{R} \& \mathrm{D}$ Systems Inc., Minneapolis, MN, USA; catalogue: 201-LB-010) for $24 \mathrm{~h}$. Cytokines were measured in basal supernatants by multiplex Luminex (EMD Milliplex, Temecula, CA, USA; catalogue: HCYTMAG-60K-PX30). Total RNA was isolated using an RNeasy Mini Kit (Qiagen, Germantown, MD, USA) and sent for standard RNA sequencing (Illumina, single end read, 50 bp, $30 \mathrm{M}$ ).

Results

Serum G-CSF is significantly elevated in a subset of patients hospitalised for exacerbations

We measured serum G-CSF during severe hospitalised exacerbation and after 30 days of recovery in available samples from the LEUKO study (cohort demographics are summarised in table 1) [14]. Serum G-CSF was increased at the time of hospitalisation compared with the 30-Day recovery sample $(\mathrm{p}<0.0001)$ (figure 1a). A strong correlation was observed between the absolute level of serum G-CSF at exacerbation 


\begin{tabular}{|c|c|c|c|}
\hline \multirow[t]{2}{*}{ Characteristic } & \multicolumn{2}{|c|}{ LEUKO cohort ${ }^{\#}$} & \multirow[t]{2}{*}{ MRC cohort } \\
\hline & Placebo & Zileuton & \\
\hline Subjects & 33 & 36 & 59 \\
\hline Age years & $63 \pm 1.7$ & $61 \pm 1.5$ & $70 \pm 9.3$ \\
\hline \multicolumn{4}{|l|}{ Gender } \\
\hline Male & $22(67)$ & $20(56)$ & $41(70)$ \\
\hline Female & $11(33)$ & $16(44)$ & $18(30)$ \\
\hline \multicolumn{4}{|l|}{ Ethnicity } \\
\hline Caucasian & $14(42)$ & $21(58)$ & $58(98)$ \\
\hline African-american & $18(54)$ & $13(36)$ & - \\
\hline Native-american & $3(9)$ & $4(11)$ & - \\
\hline Black & - & - & $1(2)$ \\
\hline Hispanic & $2(6)$ & $3(8)$ & - \\
\hline \multicolumn{4}{|l|}{ Smoking status } \\
\hline Ex-smoker & $21(64)$ & $25(70)$ & $41(70)$ \\
\hline Current smoker & $12(36)$ & $11(30)$ & $15(25)$ \\
\hline Non-smoker & - & - & $3(5)$ \\
\hline \multicolumn{4}{|l|}{ GOLD stage } \\
\hline Stage 2 & - & - & $28(47)$ \\
\hline Stage 3 & - & - & $22(37)$ \\
\hline Stage 4 & - & - & $9(15)$ \\
\hline \multicolumn{4}{|l|}{$\mathrm{FEV}_{1} \mathrm{~L}$} \\
\hline Pre-bronchodilator & $0.87 \pm 0.09$ & $0.75 \pm 0.17$ & $1.31 \pm 0.07$ \\
\hline Post-bronchodilator & $0.81 \pm 0.11$ & $1.0 \pm 0.18$ & $1.36 \pm 0.07$ \\
\hline FVC L & $2.46 \pm 0.96$ & $2.59 \pm 0.90$ & $2.67 \pm 0.09^{9}$ \\
\hline \multicolumn{4}{|l|}{$\mathrm{FEV}_{1} / \mathrm{FVC}$} \\
\hline Pre-bronchodilator & $0.39 \pm 0.04$ & $0.44 \pm 0.04$ & $0.49 \pm 0.02$ \\
\hline Post-bronchodilator & $0.47 \pm 0.05$ & $0.45 \pm 0.04$ & $0.51 \pm 0.02$ \\
\hline
\end{tabular}

and the fold change between the exacerbation and recovery samples (Spearman's correlation=0.8, $\mathrm{p}<0.0001$ ) (supplementary figure S1A).

A cut-off of a two-fold difference between G-CSF levels at exacerbation and recovery identified 21 out of 37 G-CSF-high exacerbations with a 2.5-fold higher median serum G-CSF level (figure $1 \mathrm{~b})(\mathrm{p}<0.0001)$. The fold change of G-CSF was correlated with fold change of other biomarkers of neutrophilic inflammation such as IL-8 and serum amyloid A1 (SAA1) (figure 1c). In addition, we observed that the G-CSF-high group tended to respond better to antibiotic treatment, with a shorter length of hospitalisation compared to the G-CSF-low group of patients (supplementary figures S1B and S1C), although this did not achieve statistical significance.

\section{Elevated serum G-CSF levels identify exacerbations characterised by pathogenic bacteria and neutrophilic inflammation}

To better characterise G-CSF-high exacerbations, we utilised a second available COPD exacerbation cohort which offered paired serum and sputum samples, richer longitudinal sampling and available blood and sputum cell counts. This cohort of mild to moderate exacerbations pioneered the classification of exacerbation phenotypes using unbiased clustering analysis [3]. We measured G-CSF in 74 primary exacerbation samples that were preceded by a sample collected at a stable disease visit within the preceding 3 months, as well as at 2 weeks and 6 weeks post-exacerbation (see table 1 for patient demographics). G-CSF levels were unaffected by smoking status or $\mathrm{FEV}_{1}$ ( $\mathrm{p}>0.05$ by Mann-Whitney U-test). Increased serum G-CSF was observed in a subset of exacerbations $(p<0.0001)$ (figure $2 a)$. To characterise these G-CSF-high exacerbations, we classified exacerbations by the previously published criteria of "eosinophilic", "bacterial” or "viral” (supplementary table S1) [3] and observed significantly increased serum G-CSF in non-eosinophilic and bacterial exacerbations, but not in eosinophilic or viral exacerbations (figures 2b-2e). As bacterial and viral co-infections occur during COPD exacerbations 
a)

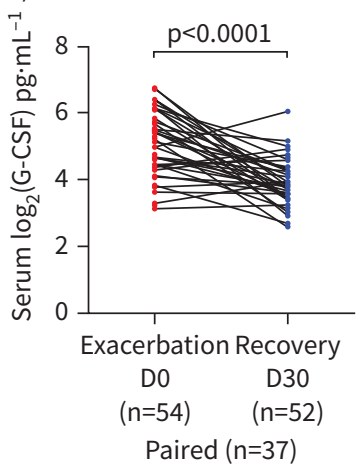

b)

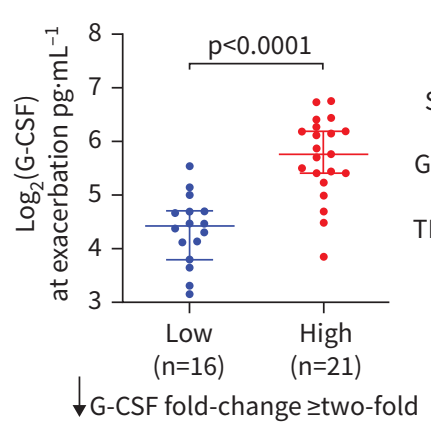

c)

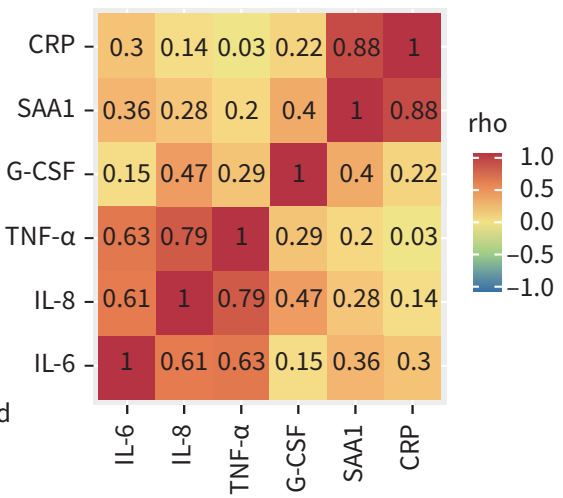

FIGURE 1 Serum granulocyte-colony stimulating factor (G-CSF) is significantly elevated during COPD exacerbation. a) Serum G-CSF was measured at exacerbation (Day0 (D0), $n=54$ ) and recovery (Day30 (D30) post-exacerbation, $n=52$ ) from a hospitalised acute exacerbation of COPD (AECOPD) cohort (the LEUKO cohort). Paired exacerbation and recovery samples were available for 37 patients and analysis was performed on pooled treatment arms as there was no obvious treatment advantage of zileuton compared with placebo. p-Values were determined by the Wilcoxon matched-pairs signed-rank test. b) Among the 37 paired samples, 21 subjects had a two-fold or greater decrease in serum G-CSF at recovery (median values $\pm 95 \%$ confidence intervals (Cls) are shown and p-values were determined by the Mann-Whitney U-test. c) A heatmap of Spearman's correlations between the fold-change of the biomarkers at exacerbation compared to a preceding stable visit. Each square represents the correlation between the feature heading the column with the feature heading the row. The number shown in a given square indicates the corresponding Spearman correlation coefficient between the two features. CRP: C-reactive protein; SAA1: serum amyloid A1; TNF- $\alpha$ : tumour necrosis factor- $\alpha$; IL: interleukin.

(supplementary table S1) [3], we also examined the subset of exacerbations with any diagnosed pathogenic bacteria or virus and also observed increased G-CSF $(\mathrm{p}<0.0001)$ (figure $2 \mathrm{f}$ ).

We then characterised the inflammatory characteristics of the twenty G-CSF-high exacerbations (defined by a two-fold or greater change in serum G-CSF between exacerbation and a preceding stable visit). G-CSF-high exacerbations showed a greater increase in median blood neutrophil count than G-CSF-low exacerbations (a 4.8-fold increase versus a 1.5-fold increase from stable disease levels) (figure 3a). They also showed a 7.5-fold higher median level at exacerbation of blood neutrophils and higher levels of IL-6 and SAA1, which promote neutrophilic inflammation (figures 3b-3d). Serum levels of other cytokines associated with bacterial (IL-8, TNF- $\alpha$, IL-1 $\beta$ ), viral (CXCL10) and Type 2 inflammation (blood eosinophil counts) did not differ between G-CSF-high and G-CSF-low exacerbations ( $\mathrm{p}>0.05$, data not shown). G-CSF-high exacerbations were also characterised by an increased sputum neutrophil percentage and sputum IL-6 level (figures 3e and 3f). Serum G-CSF at exacerbation was well correlated with multiple metrics of neutrophilic inflammation, including blood and sputum neutrophils, serum IL-6, SAA1 and C-reactive protein (CRP) (Spearman's correlations (rho) $\geqslant 0.5$ ). It was also moderately correlated with sputum IL-6 and IL-8 (Spearman's correlations (rho) $\geqslant 0.3$ ) and was not correlated with biomarkers of viral or eosinophilic inflammation (figure $3 g$ and supplementary figure S2). When fold change between stable disease and exacerbation was compared, serum G-CSF correlated with blood neutrophils, serum IL-6, SAA1 and sputum IL-6 (Spearman's correlations (rho) $\geqslant 0.4$ ) (figure 3h and supplementary figure S2). Calculating fold change between levels at exacerbation and either baseline or 6-week recovery samples, in line with the results in the LEUKO cohort, gave comparable results (data not shown).

The identification of biomarkers that could be measured during stable disease to predict exacerbation subtypes would enable patient enrichment, but has been challenging to accomplish. In a previous study of this cohort, no single biomarker measured in stable disease could predict an exacerbation phenotype well. We observed a trend that patients with higher G-CSF during stable disease were more likely to have exacerbations characterised by neutrophilic inflammation. When subjects with the highest and lowest quartile of stable disease G-CSF were compared, high stable disease G-CSF was associated with higher blood neutrophils and significantly higher sputum neutrophils, sputum IL-8 and serum G-CSF at exacerbation $(\mathrm{p}<0.05)$ (supplementary figure S3). 
a)

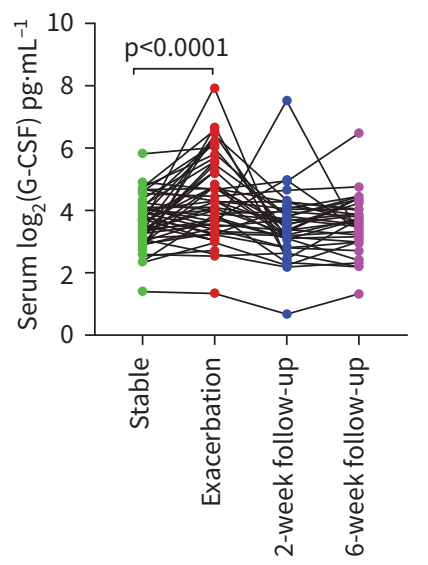

d)

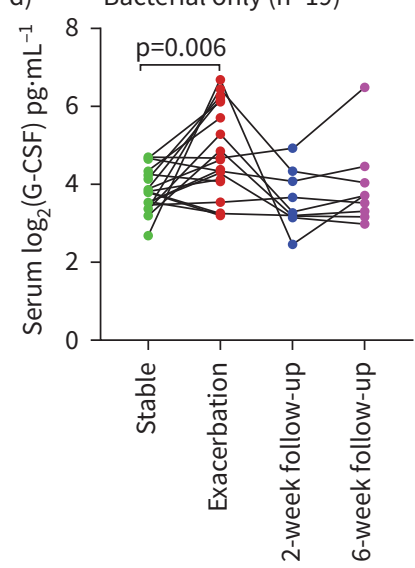

b)

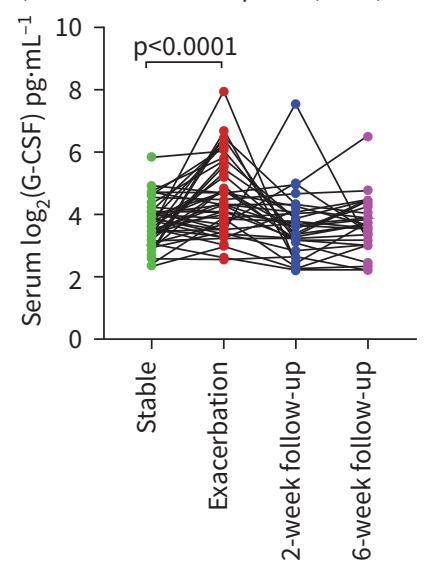

e)

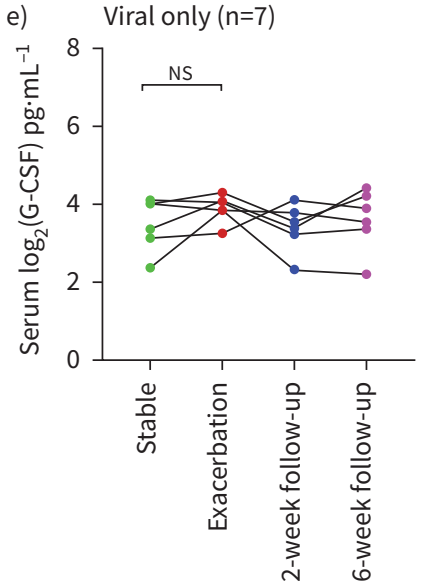

c)

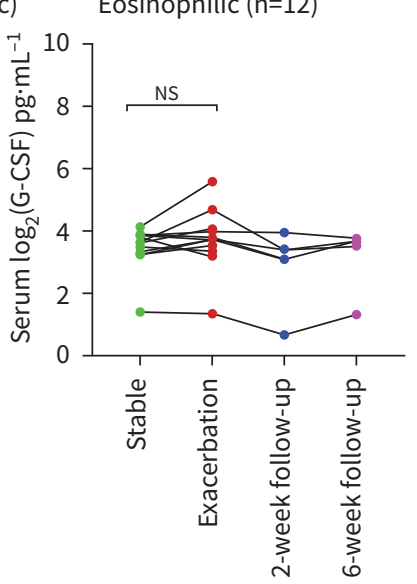

f)

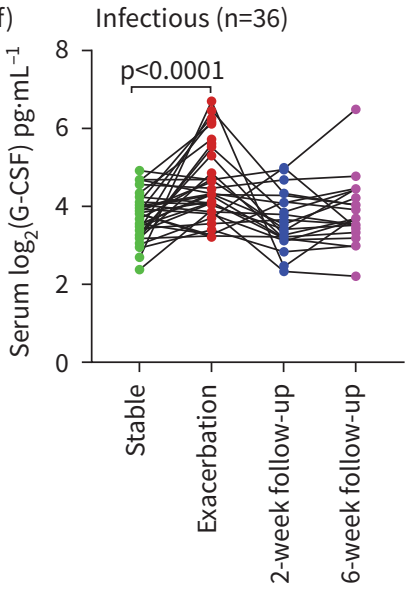

FIGURE 2 Elevated levels of serum granulocyte-colony stimulating factor (G-CSF) during exacerbation are non-eosinophilic and predominantly associated with infection. a) Serum G-CSF was measured for 74 exacerbations with preceding stable visits and at 2-week and 6-week follow-up visits for 54 subjects (the MRC cohort). Serum G-CSF measurements were categorised between b) non-eosinophilic and c) eosinophilic exacerbations. Serum G-CSF measurements were compared between exacerbations strictly associated with d) bacterial infection ( $n=19)$, e) viral infection $(n=7)$, or $f$ ) conditions described as "infectious" (bacterial, viral or bacterial/viral co-infection $(n=36))$. Statistical significance is shown by $p$-values generated by the Wilcoxon matched-pairs signed-rank test.

Elevated serum G-CSF correlates with sputum IL-1B, a key biomarker of bacterial exacerbations

Bacterial exacerbations exhibited a larger increase in G-CSF and higher absolute levels when compared with non-bacterial exacerbations (figures $4 \mathrm{a}$ and $4 \mathrm{~b}$ ). Sputum IL-1 $\beta$ is the strongest immune correlate of bacterial exacerbations (figure 4c) [3, 4, 6]. Moreover, a strong correlation of sputum IL-1 $\beta$ and serum G-CSF levels at exacerbation was observed (Spearman's correlation (rho) $=0.6, p<0.0001$ ) (figure $4 d$ ). A previous study using this cohort identified CRP as the best serum predictor of bacterial exacerbation [3]. In the subset of samples included in this study, we observed the expected higher serum levels of CRP in bacterial exacerbations and strong correlation between CRP and G-CSF (Spearman's correlation (rho)=0.7, $\mathrm{p}<0.0001$ ) (figures 4e and 4f). Performance of G-CSF in classifying bacterial exacerbations was evaluated by calculating the AUC of the ROC curve compared with blood neutrophil count and serum CRP. Serum G-CSF classified bacterial exacerbations with an AUC of the ROC curve of 0.76, which was comparable to the AUC of the ROC curve for blood neutrophils and serum CRP ( 0.70 and 0.71 , respectively) (figure $4 \mathrm{~g})$.

G-CSF-high exacerbations are preceded by an altered sputum microbiome

COPD exacerbations are associated with lung microbiome dysbiosis [19-25]. Exacerbation subtype cluster characterised by high sputum IL-1 $\beta$ and mediators of neutrophilic inflammation are enriched by the presence of potentially pathogenic bacteria such as Haemophilus and Moraxella [6, 21, 24, 25]. We 


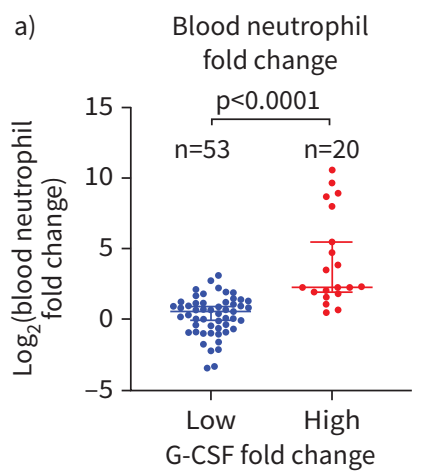

d) Serum SAA1 at exacerbation e)

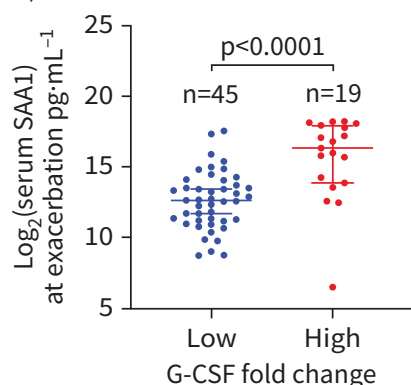

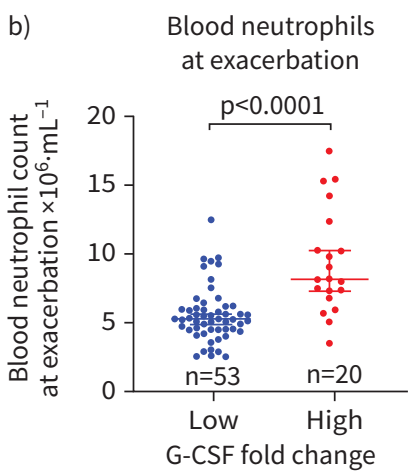

c)
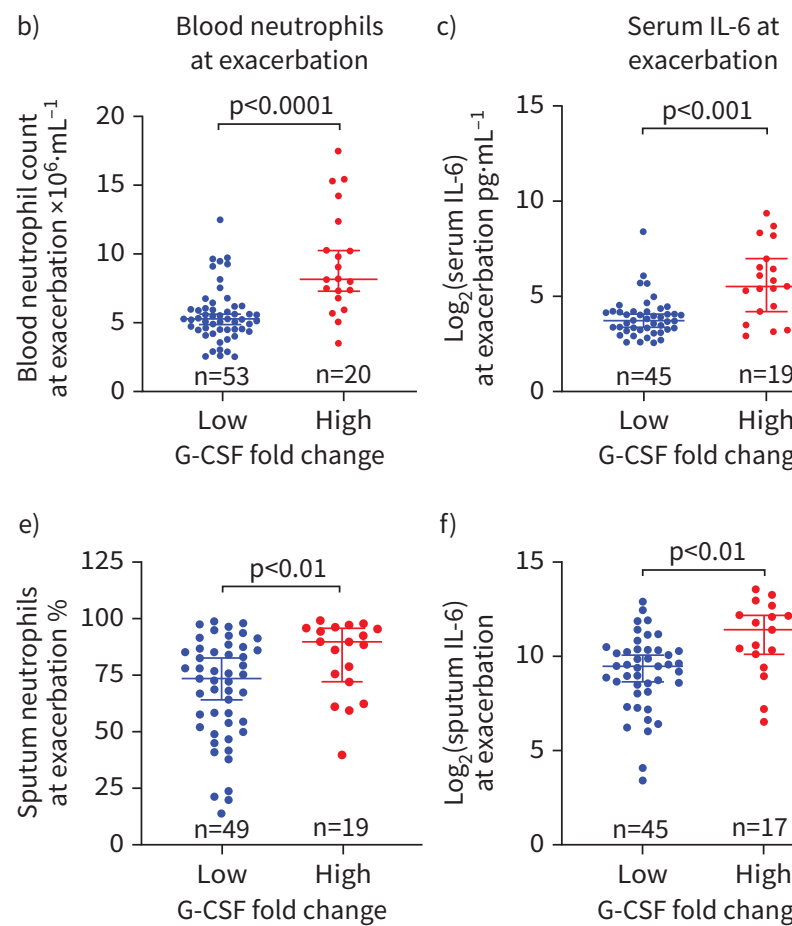

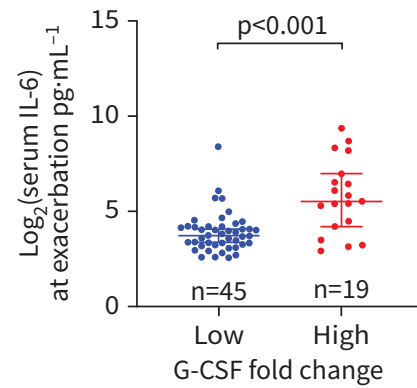

f)

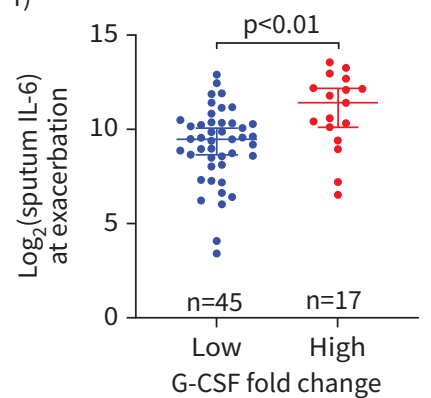

g) Correlation heatmap of biomarkers at exacerbation

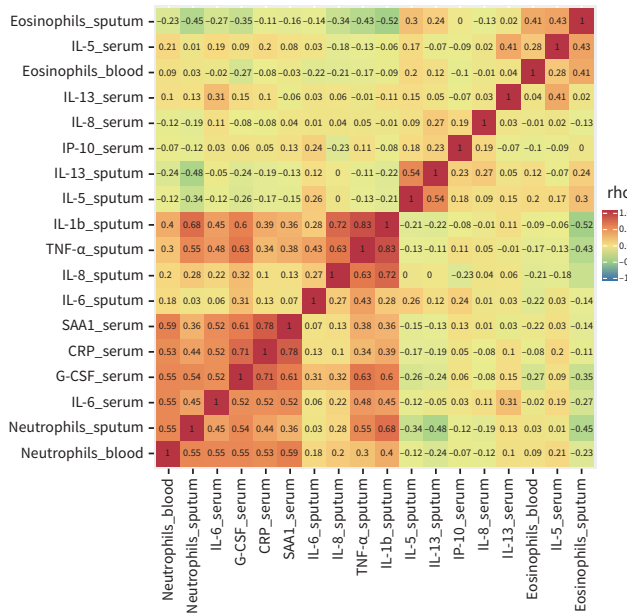

h) Correlation heatmap of biomarker fold changes Neutrophils_blood - $-0.110 .02-0.04-0.12-0.09-0.280 .270 .040 .240 .220 .160 .270 .440 .260 .370 .390 .16$

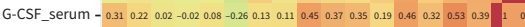
SAA1_Serum - $0.220 .23 \quad 0.190 .07-0.06-0.24-0.040 .1 \quad 0.160 .340 .3-0.030 .460 .20 .72 \quad 1$

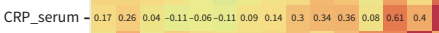
IL-5_Serum - $0.060 .37-0.020 .240 .110 .050 .10 .190 .290 .180 .320 .520 .55$ I 0.40 .20 .320 .26

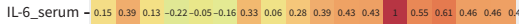

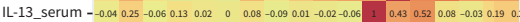

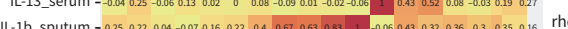

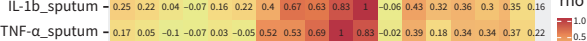

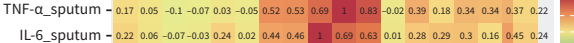

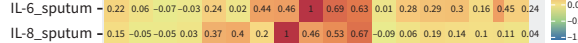
eutrophils_sputum - $-0.03-0.15-0.12-0.320 .170 .04$ 工 $0.20 .440 .520 .40 .080 .330 .110 .09-0.040 .130 .27$ Eosinophils_blood - $0.180 .05-0.040 .180 .29+0.040 .40 .02-0.050 .22 \quad 0 \quad-0.16-0.05-0.11-0.24-0.26-0.28$

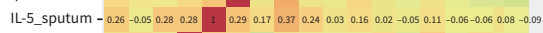
Eosinophils_sputum - $0.090 .15-0.01$ I 0.28 0.18-0.32 $0.03-0.03-0.07-0.070 .13-0.220 .24-0.110 .07-0.02-0.12$ IL-13_sputum - 0.220 .56 工 $-0.010 .28-0.04-0.12-0.05-0.07-0.10 .04-0.060 .13-0.020$ - $0.040 .190 .02-0.04$

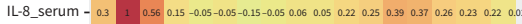
IP-10_Serum - $10.30 .220 .090 .260 .18-0.030 .150 .220 .170 .25-0.040 .150 .060 .170 .220 .31-0.11$

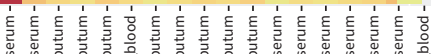

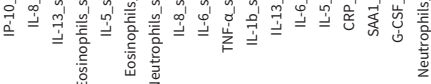

FIGURE 3 Exacerbations categorised by granulocyte-colony stimulating factor (G-CSF) induction are characterised by higher biomarkers of neutrophilic inflammation. G-CSF high and low groups are categorised based on a two-fold or higher increase in G-CSF on exacerbation compared to a preceding stable visit. a) Blood neutrophil fold-change is the change in blood neutrophils on exacerbation compared to a preceding stable visit. b) Absolute blood neutrophil count at exacerbation. c) Serum interleukin (IL)-6 at exacerbation. d) Serum amyloid A1 (SAA1) at exacerbation. e) Sputum neutrophil percentage at exacerbation. f) Sputum IL-6 at exacerbation. The p-values shown are determined by Mann-Whitney U-test. g) Heatmap of Spearman's correlations between biomarkers at exacerbation. h) Heatmap of Spearman's correlations between biomarker fold-changes at exacerbation compared to the preceding stable visit. Each square represents the correlation between the feature heading the column and the feature heading the row. The number shown in a given square indicates the corresponding Spearman correlation coefficient between the two features. IFN: interferon; IP-10: IFN- $\gamma$ induced protein-10; TNF- $\alpha$; tumour necrosis factor- $\alpha$; CRP: C-reactive protein.

hypothesised that bacterial exacerbations and exacerbations characterised by high serum G-CSF levels are preceded by an altered lung microbiome composition. To test this hypothesis, microbiome analysis was performed on available sputum from exacerbations $(n=41)$ and the preceding stable visits $(n=46)$. 


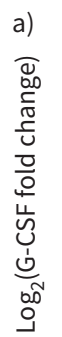

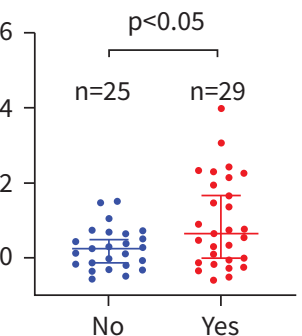

Bacterial exacerbation

c)

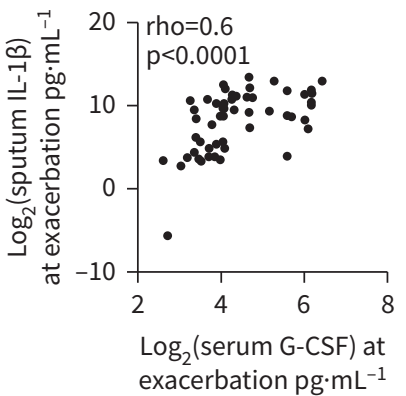

e)

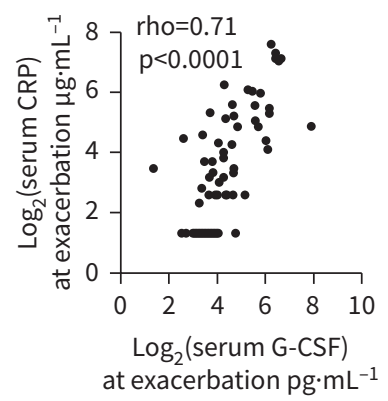

b)

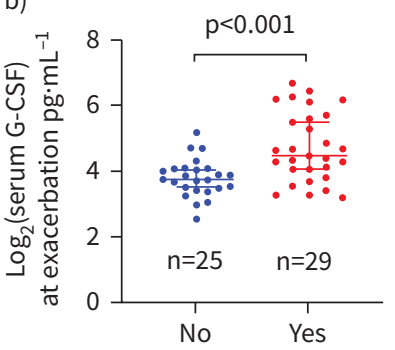

Bacterial exacerbation

d)

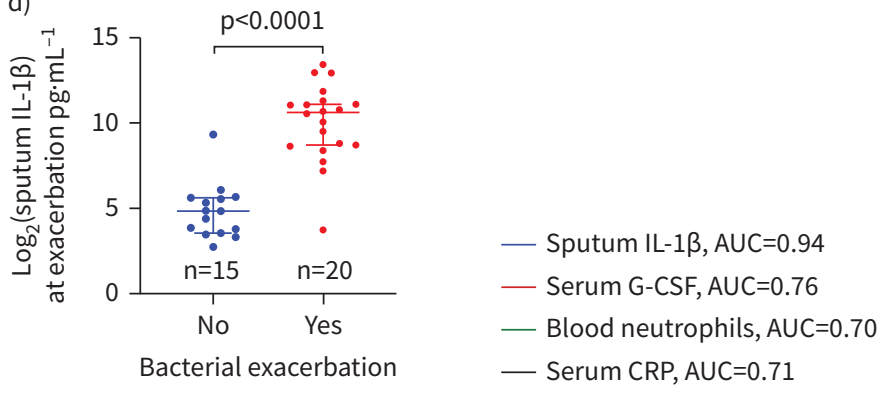

f)

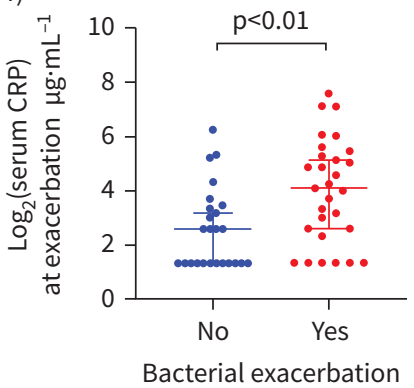

g)

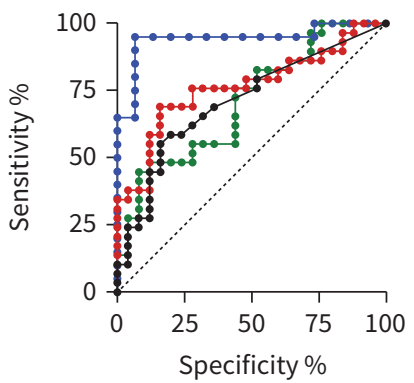

FIGURE 4 High granulocyte-colony stimulating factor (G-CSF) levels at exacerbation are associated with higher frequency of bacterial infection. Biomarker levels were compared between bacterial versus non-bacterial exacerbations. a) G-CSF fold change between exacerbation and a preceding stable visit. b) Serum G-CSF at exacerbation. c) Spearman's correlation plot of sputum interleukin (IL)- $1 \beta$ at exacerbation versus serum G-CSF at exacerbation. d) Sputum interleukin IL-1 $\beta$ at exacerbation. e) Spearman's correlation plot of serum C-reactive protein (CRP) at exacerbation versus serum G-CSF at exacerbation. f) Serum CRP at exacerbation. g) Receiver operating characteristic (ROC) curve illustrating biomarkers that positively predict bacterial exacerbation. For non-correlation plots, median values $\pm 95 \%$ confidence intervals $(\mathrm{Cls})$ are shown. $\mathrm{p}$-Values are determined by the Mann-Whitney U-test. AUC: area under the curve.

Reduced $\alpha$-diversity and a gross shift in overall sputum microbiome composition was observed prior to bacterial exacerbations (figure $5 \mathrm{a}$ and $5 \mathrm{~b}$ ). Moreover, relative abundance of the potential respiratory pathogens Haemophilus and Moraxella displayed a greater than 7.0-fold and 1.5-fold enrichment, respectively, prior to bacterial exacerbations compared to non-bacterial exacerbations, although the increase in Moraxella did not meet statistical significance after correction for false discovery rate (FDR) (figures 5c and $5 \mathrm{~d}$ ). In general, these observations persisted through exacerbation visits, with reduced $\alpha$-diversity together with Haemophilus and Moraxella enrichment also occurring in sputum samples collected during bacterial exacerbations (supplementary figures S4A-S4D). Although $\alpha$-diversity was not significantly reduced during stable visits preceding G-CSF-high exacerbations (figure 5e), an altered sputum microbiome composition (characterised by a greater than 13.0-fold enrichment of both Haemophilus and Moraxella, with only the increase in Moraxella remaining significant after FDR correction) was still observed prior to these G-CSF-high exacerbations (figures 5f-5h). However, while Moraxella enrichment 

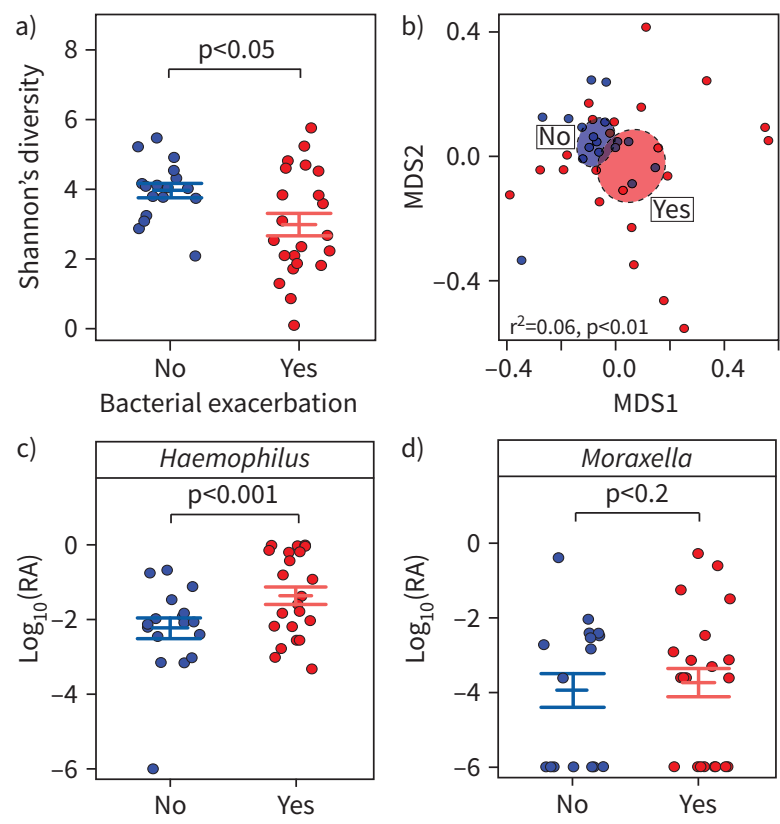

Bacterial exacerbation

Bacterial exacerbation
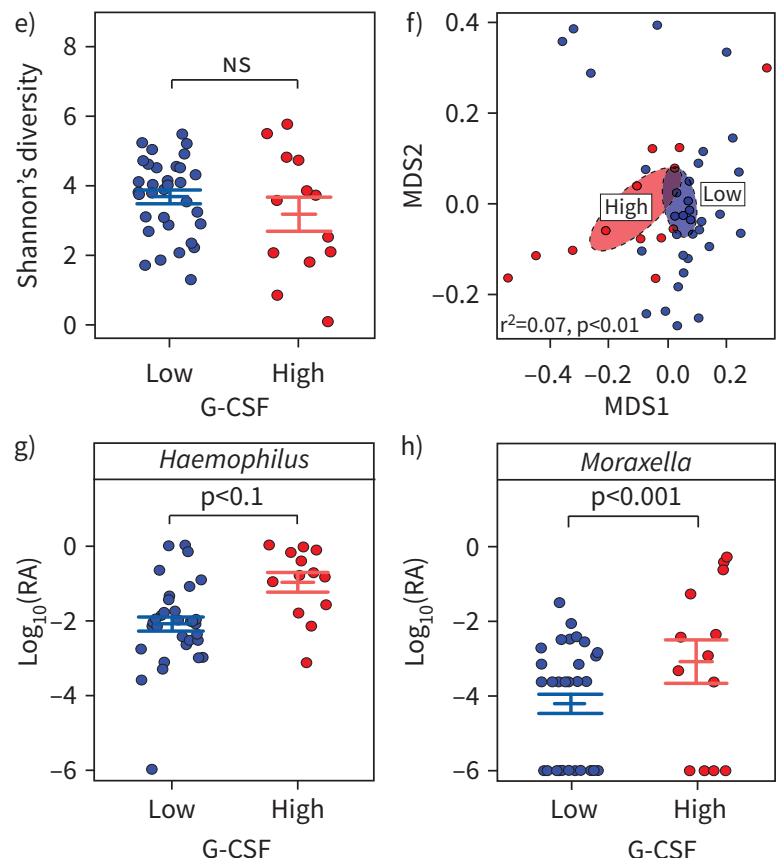

FIGURE 5 An altered sputum microbiome precedes exacerbations characterised by bacteria or a two-fold or greater increase in serum granulocyte-colony stimulating factor (G-CSF). a) Shannon's diversity index for bacterial exacerbation. b) Non-metric multi-dimensional scaling (NMDS) ordination plot of Bray-Curtis distances for bacterial exacerbation. Dashed ellipses represent the $95 \%$ confidence interval (CI) for the centroid of each stratification group. c) Haemophilus abundance for sputum microbiota during stable disease prior to bacterial exacerbation. d) Moraxella abundance for sputum microbiota during stable disease prior to bacterial exacerbation. e) Shannon's diversity index for a two-fold or greater increase in serum G-CSF. f) NMDS ordination plot of Bray-Curtis distances for a two-fold or greater increase in serum G-CSF. Dashed ellipses represent the $95 \% \mathrm{Cl}$ for the centroid of each stratification group. g) Haemophilus abundance for sputum microbiota during stable disease prior to a two-fold or greater increase in serum G-CSF. h) Moraxella abundance for sputum microbiota during stable disease prior to a two-fold or greater increase in serum G-CSF. Cohort sizes are as follows: for bacterial exacerbation (a-d), No $(n=17)$, Yes $(n=22)$. For exacerbations characterised by a two-fold or greater increase in G-CSF (e-h), Low $(n=33)$, High $(n=13)$. For panels a) and e), group mean \pm standard error ( $\mathrm{SE}$ ) values are shown and $\mathrm{p}$-values are determined by the Wilcoxon rank-sum test. 
For panels b) and (f), permutational ANOVA was calculated. For panels c), d), g) and h), the $y$-axes have been fixed to the limits of $\log _{10}(\mathrm{RA})$ for these samples, group mean $\log _{10}(\mathrm{RA}) \pm \mathrm{SE}$ values are depicted and statistical significance is determined by DESeq2 and adjusted for false discoveries. RA: relative abundance.

persisted in sputum collected during G-CSF-high exacerbations, Haemophilus abundance was slightly reduced (supplementary figures S4E-S4H).

a)

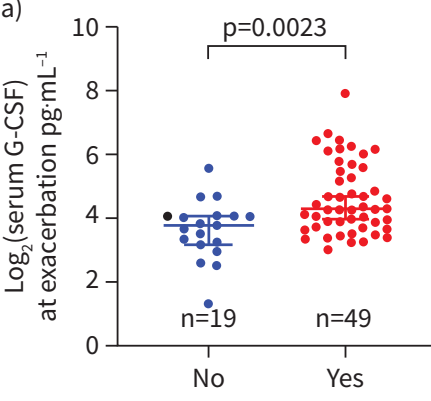

Neutrophil - high COPD at exacerbation

c)

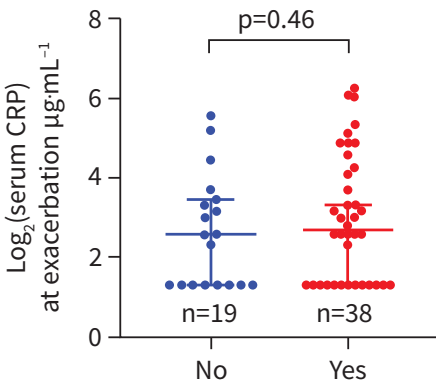

Neutrophil - high COPD at exacerbation

e)

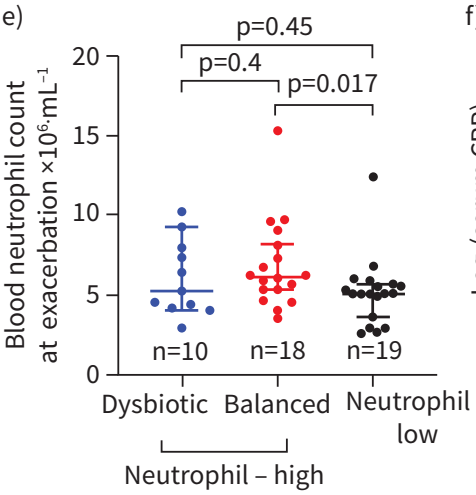

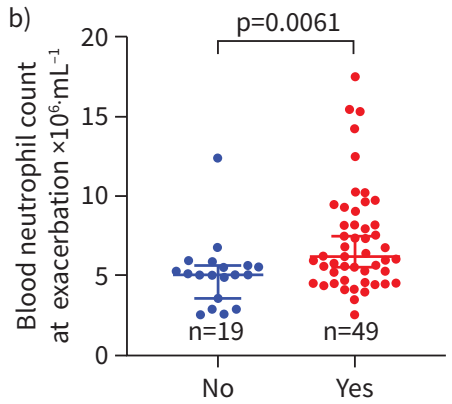

Neutrophil - high COPD at exacerbation

d)

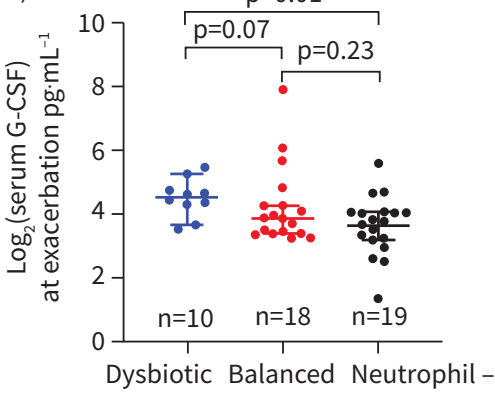

Neutrophil - high

low

Serum G-CSF, AUC $=0.75$

g)

Blood neutrophils, $A U C=0.50$

f)
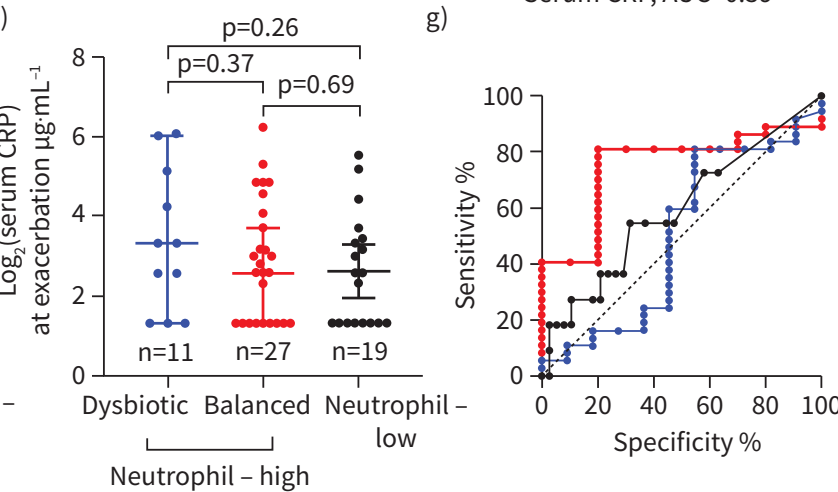

FIGURE 6 Serum granulocyte-colony stimulating factor (G-CSF) levels at exacerbation stratify neutrophil-dysbiotic exacerbations. a) Serum G-CSF, b) blood neutrophil and c) serum C-reactive protein (CRP) levels at exacerbation were compared for neutrophil and neutrophil-high (sputum neutrophils $\geqslant 61 \%$ ) COPD exacerbations. d) Serum G-CSF, e) blood neutrophil and f) serum CRP levels at exacerbation were also compared between neutrophil-dysbiotic COPD exacerbations (relative abundance of Haemophilus and Moraxella $>0.41$ ), neutrophil-balanced and neutrophil-low (sputum neutrophils <61\%) COPD exacerbations. A receiver operating characteristic (ROC) curve g) was also produced to classify neutrophil-dysbiotic COPD exacerbations. p-Values were determined by the Mann-Whitney U-test. AUC: area under the curve. 
High serum G-CSF levels at exacerbation characterise a neutrophilic-dysbiotic phenotype

A recent report has shown that two major types of airway ecology exist for neutrophilic COPD and these are differentiated by the predominance of Haemophilus [10]. Accordingly, we examined whether high serum G-CSF levels were associated with the exacerbation endotype characterised by high sputum neutrophils and overabundance of potentially pathogenic organisms such as Haemophilus and/or Moraxella. We found that serum G-CSF and blood neutrophil counts, but not serum CRP, were significantly higher in neutrophilic COPD exacerbations (as defined by sputum neutrophils $>61 \%$ ) (figures 6a-6c). Next, we stratified the neutrophilic COPD exacerbations into neutrophilic-dysbiotic and neutrophilic-balanced exacerbations based on a combined RA of Haemophilus and Moraxella of $>0.41$. Median serum G-CSF was significantly higher in neutrophilic-dysbiotic COPD exacerbations compared to the neutrophil-low COPD exacerbations, while blood neutrophil counts and serum CRP levels were not. Median G-CSF level trended higher in neutrophilic-dysbiotic exacerbations compared to neutrophilic-balanced exacerbations but this was not significant $(p=0.07$ ) (figures $6 \mathrm{~d}-6 \mathrm{f}$ ). Performance of G-CSF in classifying neutrophilic-dysbiotic exacerbations was evaluated by calculating the AUC of the ROC curve compared with blood neutrophils and serum CRP. Serum G-CSF stratified neutrophilic-dysbiotic COPD with an AUC of the ROC curve of 0.75, while blood neutrophil count and serum CRP had values of 0.50 and 0.59 , respectively (figure $6 \mathrm{~g}$ ).
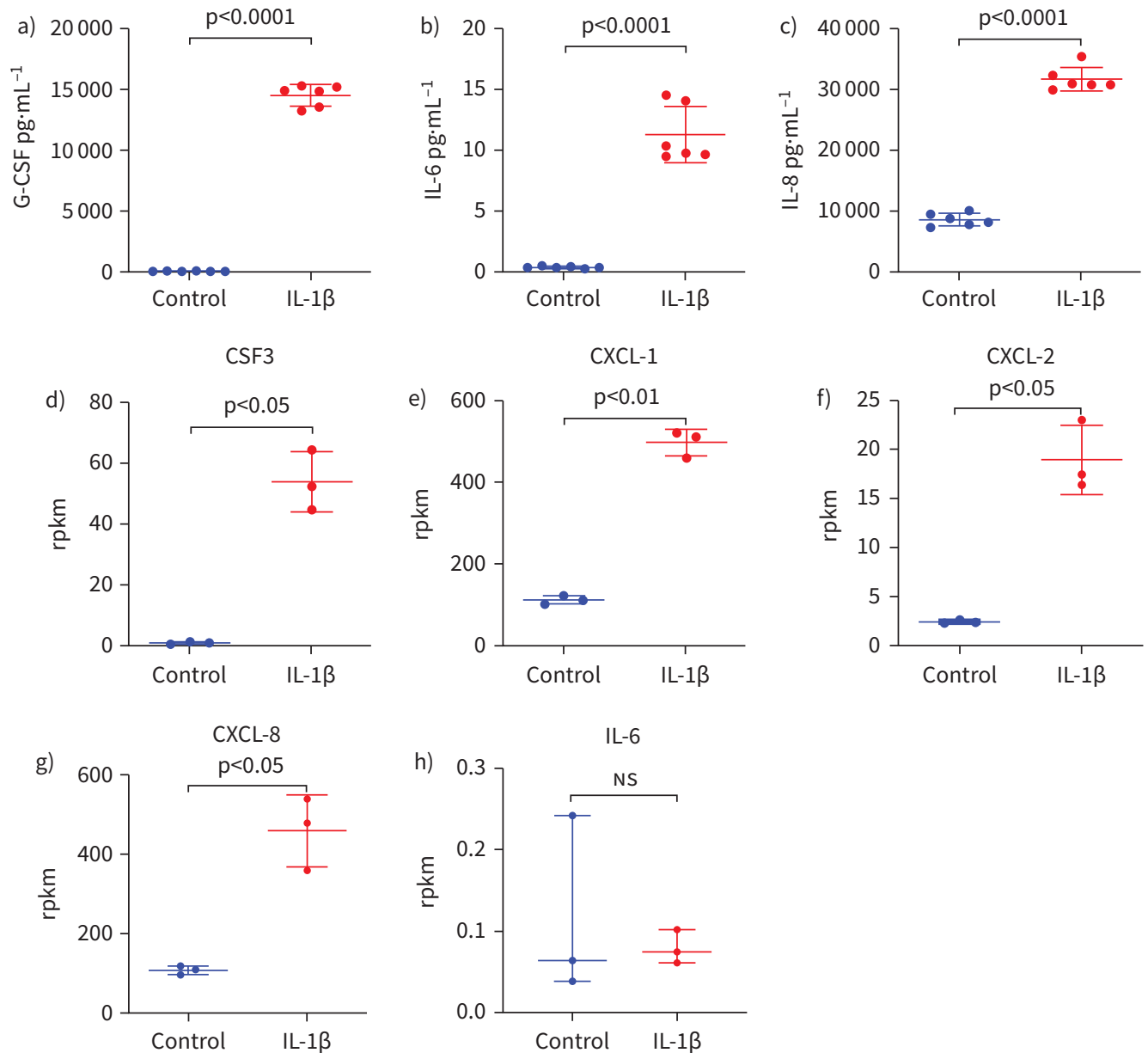

FIGURE 7 Interleukin (IL)-1 $\beta$ treatment directly induces genes conducive of neutrophilic inflammation. Normal human bronchial epithelial cells were differentiated at the air-liquid interface and treated with IL-1 $\beta$ $\left(10 \mathrm{ng} \cdot \mathrm{mL}^{-1}\right)$ for $24 \mathrm{~h}$. Cytokine levels were then measured from cell supernatants by Luminex-based enzyme-linked immunosorbent assay (ELISA) ( $\mathrm{a}-\mathrm{c})$. RNA was also isolated and gene expression was examined by RNA sequencing $(d-h)$. Statistical significance was determined by the unpaired t-test with Welch's correction. CSF: colony stimulating factor; CXCL: chemokine (C-X-C motif) ligand. rpkm: reads per kb transcript per million mapped reads. 
Treatment of primary lung epithelial cells with IL-1B induces G-CSF production

We were interested in the mechanistic connection between the module of neutrophil-associated biomarkers correlating with G-CSF. An IL-1 $\beta$ response signature in lung epithelial cells was previously identified, which included G-CSF, IL-6 and TNF- $\alpha$ [6]. We confirmed that IL-1 $\beta$ was sufficient to induce G-CSF and IL-6 secretion from primary bronchial epithelial cells cultured at the air-liquid interface (figures $7 \mathrm{a}$ and $7 b)$. IL-1 $\beta$ also increased transcription of multiple neutrophil-recruiting chemokines (CXCL1, CXCL2 and CXCL8/IL-8) and secretion of IL-8 (figures 7c-7g). However, transcription of IL-6 was not induced by

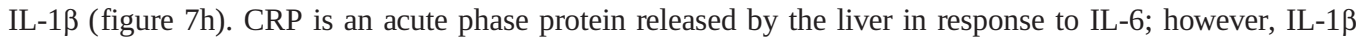
did not induce transcription of CRP by lung epithelial cells (data not shown). Therefore, systemic G-CSF might be mechanistically connected to lung neutrophilic response to dysbiosis orchestrated by local production of IL-1 $\beta$.

\section{Discussion}

A better understanding of the molecular drivers of exacerbation phenotypes is needed to develop targeted therapeutic interventions for COPD patients. Biomarker classifiers are needed to study exacerbation heterogeneity in observational patient cohorts and to interpret data from clinical trials. This exploratory study identifies G-CSF as a novel molecular feature of the bacterial/neutrophilic exacerbation subtype described by other groups [3, 4, 6, 10]. Serum G-CSF classifies neutrophilic-dysbiotic exacerbations better than serum CRP or blood neutrophils. This recently described exacerbation subtype is characterised by high sputum neutrophils and IL-1 $\beta$, as well as an abundance of potential bacterial pathogens such as Haemophilus and Moraxella identified by $16 \mathrm{~S}$ sequencing (supplementary figure S5) [10]. Serum G-CSF correlates with neutrophils and neutrophil-promoting factors, such as IL-6 and IL-8, in the lung (as sampled by induced sputum during exacerbation) and in the circulation. Serum G-CSF correlates with sputum IL-1 $\beta$ and we have demonstrated, using physiologically-relevant primary differentiated bronchial epithelial cells, that IL-1 $\beta$ stimulation is sufficient for release of G-CSF, IL-6 and IL-8. G-CSF has pleiotropic effects on neutrophils, which are a predominant inflammatory cell type in the lungs of many COPD patients [13, 26-28]. It promotes granulopoiesis and inhibits apoptosis, which together contribute to increased neutrophil numbers in the lung [26, 27, 29, 30]. G-CSF can also alter neutrophil functionality such as chemotaxis, reactive oxygen species production and degranulation [31, 32]. How this may contribute to impaired response to infection and exacerbation severity in COPD patients is an interesting area for further investigation.

Predictors of exacerbation subtype that could be measured during stable disease would greatly facilitate patient selection into clinical trials of targeted therapies. Our data suggests that the increased frequency of bacterial aetiology in exacerbations associated with bacterial infection and G-CSF-high exacerbations may be related to a preceding dysbiosis in the lung microbiome. Although earlier reports highlighted microbial dysbiosis during exacerbation, this is the first instance where a preceding stable state is shown to be enriched with potentially pathogenic respiratory bacteria and associated with exacerbation subtypes defined by bacterial infection or high serum G-CSF. Moreover, subtypes of COPD are predicted to have distinct lung microbiome compositions and stabilities over time [19], which highlights the importance of better understanding the relationship of the lung microbiome during stable disease to exacerbation phenotypes from both biomarker and mechanistic perspectives.

We also observed a trend that patients with high serum G-CSF measured during stable disease were more likely to have exacerbations characterised by neutrophilic inflammation (supplementary figure S3). The relationships between stable disease levels of serum G-CSF, lung neutrophilic inflammation and microbial dysbiosis, and exacerbation phenotypes requires characterisation in larger cohorts.

Limitations of this study include the small number of samples available for exacerbation subtypes and the limited size of the microbiome dataset. Validation studies are underway to evaluate G-CSF in additional cohorts. The trend that patients with G-CSF-high exacerbations responded better to antibiotic therapy, with a shorter duration of hospitalisation observed in the LEUKO cohort, requires validation in larger independent cohorts. The MRC cohort did not sample all exacerbations, precluding an analysis of the stability of G-CSF-high exacerbations in patients and examination of prognostic biomarkers for the rate of G-CSF-high exacerbations. The viral and bacterial PCR panels were not comprehensive, and as such viral and bacterial exacerbations were potentially under-diagnosed. Applying metagenomics approaches in future studies will yield a more comprehensive view of relationships between microbes and host inflammatory responses.

Cumulatively, our data suggest that COPD patients having bacterial exacerbations are associated with dysbiotic lung microbiome at a preceding stable state (with lower microbial diversity driven by increased 
abundance of respiratory pathogens like Haemophilus and Moraxella). This exacerbation endotype is associated with an overabundance of bacterial load in the lung, reflected by high levels of sputum IL-1 1 , neutrophils and serum G-CSF. Accordingly, confirming serum G-CSF as a prospective biomarker for neutrophilic-dysbiotic exacerbations in additional cohorts will aid in enriching subjects for precision medicine.

Acknowledgements: We thank John E. Connett (University of Minnesota), Prescott G. Woodruff (University of California San Francisco) and other COPD Clinical Research Network (CCRN) collaborators for sharing samples and data from the LEUKO study. We thank Christopher E. Brightling (University of Leicester) and Mona Bafadhel (University of Oxford) for sharing samples and data from the MRC cohort. We thank Biorender for their help with illustration.

Data availability: This secondary analysis of two previously described observational cohorts was performed in collaboration with the study investigators and in concordance with our data sharing agreement with them. This study makes new biomarker measurements on archived samples. Key investigators who contributed to the current study are recognised as coauthors.

Conflict of interest: A. Chakrabarti reports personal fees from and stock in Genentech, Inc., during the conduct of the study and outside the submitted work. J.S. Mar reports personal fees from and equity in Genentech, Inc., outside the submitted work. D.F. Choy is an employee of Genentech, Inc. Y. Cao reports personal fees from and stock in Genentech, Inc., during the conduct of the study and outside the submitted work. N. Rathore reports personal fees from and stock in Genentech, Inc., during the conduct of the study and outside the submitted work. X. Yang reports personal fees from Genentech, Inc., during the conduct of the study and outside the submitted work. G.W. Tew reports personal fees from Genentech, Inc., during the conduct of the study and outside the submitted work. O. Li is a salaried employee of Genentech, Inc. and received nonfinancial support from Genentech, Inc., during the conduct of the study. P.G. Woodruff reports consulting fees from Regeneron, Sanofi, Glenmark Pharma, Theravance and NGM Pharma, and visiting professor honoraria from Amgen and Genentech, outside the submitted work. C.E. Brightling is an employee of the University of Leicester. M. Grimbaldeston is an employee of Genentech, Inc., and reports personal fees and nonfinancial support during the conduct of the study. S.A. Christenson reports consulting fees from AstraZeneca, GlaxoSmithKline, Amgen and Glenmark; personal fees for invited lectures from Sunovion and Genentech; and personal fees for writing for UpToDate, all outside the submitted work. M. Bafadhel reports grants from AstraZeneca; honoraria for consulting and advisory boards, as well as travel to conferences, from AstraZeneca, Chiesi and GSK. She is a scientific advisor to and minor shareholder in AlbusHealth, all outside the submitted work. C.M. Rosenberger reports personal fees from and stock in Genentech, Inc., during the conduct of the study and outside the submitted work.

Support statement: This work was supported by Genentech, Inc. Funding information for this article has been deposited with the Crossref Funder Registry.

\section{References}

1 Vogelmeier CF, Criner GJ, Martínez FJ, et al. Erratum to "Global Strategy for the Diagnosis, Management, and Prevention of Chronic Obstructive Lung Disease 2017 Report: GOLD Executive Summary" [Arch Bronconeumol. 2017; 53:128-49]. Arch Bronconeumol 2017; 53: 411-412.

2 Wedzicha JA, Wilkinson T. Impact of chronic obstructive pulmonary disease exacerbations on patients and payers. Proc Am Thorac Soc 2006; 3: 218-221.

3 Bafadhel M, McKenna S, Terry S, et al. Acute exacerbations of chronic obstructive pulmonary disease: identification of biologic clusters and their biomarkers. Am J Respir Crit Care Med 2011; 184: 662-671.

4 Ghebre MA, Bafadhel M, Desai D, et al. Biological clustering supports both "Dutch" and "British" hypotheses of asthma and chronic obstructive pulmonary disease. J Allergy Clin Immunol 2015; 135: 63-72.

5 Mathioudakis AG, Janssens W, Sivapalan P, et al. Acute exacerbations of chronic obstructive pulmonary disease: in search of diagnostic biomarkers and treatable traits. Thorax 2020; 75: 520-527.

6 Damera G, Pham TH, Zhang J, et al. A sputum proteomic signature that associates with increased IL-1 $\beta$ levels and bacterial exacerbations of COPD. Lung 2016; 194: 363-369.

7 Jurcevic S, Humfrey C, Uddin M, et al. The effect of a selective CXCR2 antagonist (AZD5069) on human blood neutrophil count and innate immune functions. Br J Clin Pharmacol 2015; 80: 1324-1336.

8 Lazaar AL, Miller BE, Tabberer M, et al. Effect of the CXCR2 antagonist danirixin on symptoms and health status in COPD. Eur Respir J 2018; 52: 1801020.

9 Thulborn SJ, Mistry V, Brightling CE, et al. Neutrophil elastase as a biomarker for bacterial infection in COPD. Respir Res 2019; 20: 170. 
10 Wang Z, Locantore N, Haldar K, et al. Inflammatory endotype associated airway microbiome in COPD clinical stability and exacerbations - a multi-cohort longitudinal analysis. Am J Respir Crit Care Med 2020; in press [https://doi.org/doi: 10.1164/rccm.202009-34480C].

11 Wiedermann FJ, Mayr AJ, Kaneider NC, et al. Alveolar granulocyte colony-stimulating factor and $\alpha$-chemokines in relation to serum levels, pulmonary neutrophilia, and severity of lung injury in ARDS. Chest 2004; 125: 212-219.

12 Wiedermann FJ, Mayr AJ, Hobisch-Hagen P, et al. Association of endogenous G-CSF with anti-inflammatory mediators in patients with acute respiratory distress syndrome. J Interf Cytokine Res 2003; 23: 729-736.

13 Aggarwal A, Baker CS, Evans TW, et al. G-CSF and IL-8 but not GM-CSF correlate with severity of pulmonary neutrophilia in acute respiratory distress syndrome. Eur Respir J 2000; 15: 895-901.

14 Woodruff PG, Albert RK, Bailey WC, et al. Randomised trial of zileuton for treatment of COPD exacerbations requiring hospitalization. COPD J Chronic Obstr Pulm Dis 2011; 8: 21-29.

15 Caporaso JG, Kuczynski J, Stombaugh J, et al. QIIME allows analysis of high-throughput community sequencing data. Nat Methods 2010; 7: 335-336.

16 Kopylova E, Navas-molina JA, Mercier C, et al. Open-source sequence clustering methods improve the state of the art. mSystems 2016; 1: e00003-15.

17 McMurdie PJ, Holmes S. Waste not, want not: why rarefying microbiome data is inadmissible. PLoS Comput Biol 2014; 10: e1003531.

18 Haynes W. Benjamini-Hochberg method. In: Dubitzky W, Wolkenhauer O, Cho K-H, et al., eds. Encyclopedia of Systems Biology. New York, Springer, 2013; p. 78.

19 Mayhew D, Devos N, Lambert C, et al. Longitudinal profiling of the lung microbiome in the AERIS study demonstrates repeatability of bacterial and eosinophilic COPD exacerbations. Thorax 2018; 73: 422-430.

20 Wang Z, Singh R, Miller BE, et al. Sputum microbiome temporal variability and dysbiosis in chronic obstructive pulmonary disease exacerbations: an analysis of the COPDMAP study. Thorax 2018; 73: 331-338.

21 Ghebre MA, Pang PH, Diver S, et al. Biological exacerbation clusters demonstrate asthma and chronic obstructive pulmonary disease overlap with distinct mediator and microbiome profiles. J Allergy Clin Immunol 2018; 141: 2027-2036.

22 Wang Z, Bafadhel M, Haldar K, et al. Lung microbiome dynamics in COPD exacerbations. Eur Respir J 2016; 47: 1082-1092.

23 Huang YJ, Sethi S, Murphy T, et al. Airway microbiome dynamics in exacerbations of chronic obstructive pulmonary disease. J Clin Microbiol 2014; 52: 2813-2823.

24 Keir HR, Dicker A, Lonergan M, et al. Clinical endotypes of exacerbation are associated with differences in microbial composition and diversity in COPD. Eur Respir J 2020; 56: 4931.

25 Dicker AJ, Crichton ML, Pumphrey EG, et al. Neutrophil extracellular traps are associated with disease severity and microbiota diversity in patients with chronic obstructive pulmonary disease. J Allergy Clin Immunol 2018; 141: 117-127.

26 Bajrami B, Zhu H, Kwak HJ, et al. G-CSF maintains controlled neutrophil mobilization during acute inflammation by negatively regulating CXCR2 signaling. J Exp Med 2016; 213: 1999-2018.

27 Semerad CL, Liu F, Gregory AD, et al. G-CSF is an essential regulator of neutrophil trafficking from the bone marrow to the blood. Immunity 2002; 17: 413-423.

28 Hoenderdos $\mathrm{K}$, Condliffe A. The neutrophil in chronic obstructive pulmonary disease: too little, too late or too much, too soon? Am J Respir Cell Mol Biol 2013; 48: 531-539.

29 Matute-Bello G, Lues WC, Radella F, et al. Neutrophil apoptosis in the acute respiratory distress syndrome. Pneumologie 1998; 52: 354-355.

30 Van Raam BJ, Drewniak A, Groenewold V, et al. Granulocyte colony-stimulating factor delays neutrophil apoptosis by inhibition of calpains upstream of caspase-3. Blood 2008; 112: 2046-2054.

31 Salzer AT, Niemiec MJ, Hosseinzadeh A, et al. Assessment of neutrophil chemotaxis upon G-CSF treatment of healthy stem cell donors and in allogeneic transplant recipients. Front Immunol 2018; 9: 1.

32 Castellani S, D'Oria S, Diana A, et al. G-CSF and GM-CSF modify neutrophil functions at concentrations found in cystic fibrosis. Sci Rep 2019; 9: 12937. 